\title{
Spectral and Scattering Theory for the Klein-Gordon Equation
}

\author{
Lars-Erik Lundberg \\ Nordita, Copenhagen \\ Received February 5, 1973
}

\begin{abstract}
Eigenfunction expansions associated with the Klein-Gordon equation, are derived in the static external field case. By employing these, we develop spectral and scattering theory. The results are almost as strong as those obtained in the Schrödinger case.
\end{abstract}

\section{Introduction}

We shall in this paper consider spectral and scattering theory for the Klein-Gordon (K-G) equation

$$
\left(\square+2 i q_{0}(x) \partial_{t}-q_{0}(x)^{2}+q_{s}(x)+m^{2}\right) u(x, t)=0,
$$

where $x \in R^{3}, t \in R$ and $\square=\partial_{t}^{2}-\Delta$. The functions $q_{0}(x)$ and $q_{s}(x)$ are static external potentials coupled like a zero'th component of a fourvector and a scalar respectively.

Equations similar to (1.1) have been studied previously. Thoe [1] developed spectral and scattering theory in the case $m=0, q_{0}(x)=0$ and $q_{s}(x)>0$ by employing a method due to Lax and Phillips [2] (only suited for the $m=0$ case). Strauss [3] showed the existence and boundedness of the scattering operator and its inverse when $m>0$ and $q_{0}(x)=0$. Veselic [4] considered some spectral properties of Eq. (1.1) in the case $m>0, q_{s}(x)=0$ under very restrictive conditions on $q_{0}(x)$ (excluding for example the square well case).

We shall consider Eq. (1.1) when $m>0$ and derive eigenfunction expansions. These will enable us to develop the spectral theory of Eq. (1.1) in detail and develop scattering theory to the same level as is possible in the Schrödinger case [5-8].

The main motivation for this investigation (from a physical point of view) is the fact that once we have developed spectral and scattering theory for Eq. (1.1) when considered as a classical field equation, the associated quantum field theoretic problem can be completely solved (and will be considered elsewhere).

In Section 2 we start by specifying the class of potentials we shall consider. Furthermore, we write the K-G equation in the form $i \partial_{t} \Psi=A \Psi$ 
and construct a Hilbert space $\mathscr{H}$ associated with it (by employing the field-energy as norm). The operator $A$ is then shown to be self-adjoint and its essential spectrum $\sigma_{\mathrm{e}}(A)$ is determined (Section 3). Eigenfunction expansions associated with $A$ are derived and the absolutely continuous spectrum $\sigma_{\text {a.c. }}(A)$ is determined (Section 4).

Finally, the existence and completeness of the wave-operators $W_{ \pm}$ are established and the explicit form of the $S$-matrix is given (Section 5).

\section{The K-G Equation Written as $i \partial_{t} \Psi=A \Psi$ and the Hilbert Space $\mathscr{H}$ Associated with it}

We shall consider Eq. (1.1) under the following conditions on the potentials $q_{0}(x)$ and $q_{s}(x)$;

i) $q_{0}(x)$ and $q_{s}(x)$ are real-valued and locally Hölder-continuous except at a finite number of singularities;

ii) $q_{0}(x)^{2}$ and $q_{s}(x)$ are square integrable;

iii) $q_{0}(x)$ and $q_{s}(x)$ behave as $\mathcal{O}\left(|x|^{-3-\varepsilon}\right), \varepsilon>0$ for $|x| \rightarrow \infty$;

iv) $\int d x\left(-q_{0}(x)^{2}+q_{s}(x)\right)|f(x)|^{2} \geqq-\alpha \int d x\left(|\nabla f(x)|^{2}+m^{2}|f(x)|^{2}\right)$, with $0<\alpha<1$ and $f(x) \in C_{0}^{\infty}\left(R^{3}\right)$.

Provided Eq. (1.1) together with $\left\{u(x, 0), i \partial_{t} u(x, 0)\right\}=\left\{f_{1}(x), f_{2}(x)\right\}$ defines a well-posed initial-value problem, it is well known that the field energy (energy integral)

$$
E\left(u, i \partial_{t} u\right)=\int d x\left(|\nabla u|^{2}+\left(m^{2}-q_{0}^{2}+q_{s}\right)|u|^{2}+\left|\partial_{t} u\right|^{2}\right),
$$

is independent of $t$ (if it is finite). Note that condition iv) on $q_{0}(x)$ and $q_{s}(x)$ ensures the positivity of $E\left(u, i \partial_{t} u\right)$.

Let us introduce the pair

$$
\Psi(x, t)=\left\{u(x, t), i \partial_{t} u(x, t)\right\},
$$

and the time evolution operator $U(t) ; \Psi(\cdot, t)=U(t) \Psi(\cdot, 0)$.

The operator $U(t)$ is easily seen to be unitary in the Hilbert space $\mathscr{H}$ consisting of initial data $f=\left\{f_{1}, f_{2}\right\}$ such that $\|f\|^{2}=E\left(f_{1}, f_{2}\right)<\infty$, provided the initial-value problem is well-posed.

Definition 2.1. Let $\mathscr{H}$ denote the Hilbert space obtained by completing $\mathscr{D}=C_{0}^{\infty}\left(R^{3}\right) \times C_{0}^{\infty}\left(R^{3}\right)$ in the norm given by

$$
\|f\|^{2}=\int d x\left(\left|\nabla f_{1}\right|^{2}+\left(m^{2}-q_{0}^{2}+q_{s}\right)\left|f_{1}\right|^{2}+\left|f_{2}\right|^{2}\right),
$$

and with the scalar product defined in the obvious way. Let furthermore $\mathscr{H}_{0}$ denote $\mathscr{H}$ when $q_{0}=q_{s}=0$. 
Remark 2.2. The norms in $\mathscr{H}_{0}$ and $\mathscr{H}$ are equivalent (this follows from condition ii) and iv) on the potentials), i.e. there exist constants $c_{1}$ and $c_{2}$ such that

$$
0<c_{1}\|f\|_{0} \leqq\|f\| \leqq c_{2}\|f\|_{0},
$$

where \|\|$_{0}$ denotes the norm in $\mathscr{H}_{0}$.

The K-G equation (1.1) can be written

where

$$
i \partial_{t} \Psi=A \Psi
$$

$$
A=\left(\begin{array}{cc}
0 & 1 \\
L & 2 q_{0}
\end{array}\right), \quad L=-\Delta+m^{2}+q, \quad q=-q_{0}^{2}+q_{s},
$$

and with $\Psi$ given by Eq. (2.2). One can easily check that $A$ is symmetric on $\mathscr{D}$ in $\mathscr{H}$.

In the next section we shall prove that $A$ is essentially self-adjoint on $\mathscr{D}$ in $\mathscr{H}$ and furthermore determine the essential spectrum of its closure (also denoted by $A$ ) in $\mathscr{H}$.

\section{Self-adjointness of $A$ in $\mathscr{H}$ and the Essential Spectrum $\sigma_{e}(A)$}

In this section we show that $A$ is essentially self-adjoint on $\mathscr{D}$ in $\mathscr{H}$ by using a well-known theorem of Kato and Rellich (generalized to the case of two Hilbert spaces by Thoe [1]). We furthermore determine the essential spectrum of $A\left(\sigma_{\mathrm{e}}(A)\right)$ in $\mathscr{H}$, by a compactness argument.

The operator $A$ can be split into two parts

where

$$
A=A_{0}+V \text {, }
$$

$$
A_{0}=\left(\begin{array}{cc}
0 & 1 \\
L_{0} & 0
\end{array}\right), \quad V=\left(\begin{array}{cc}
0 & 0 \\
q & 2 q_{0}
\end{array}\right), \quad L_{0}=-\Delta+m^{2} .
$$

Let $H^{n}$ denote the Sobolev space consisting of all functions, which together with their derivatives up to order $n$, are square integrable.

We note that $\mathscr{H}=\mathscr{H}_{0}=H^{1} \times H^{0}=H^{1} \times L^{2}\left(R^{3}\right)$. Let us start with the following well-known results (when $m>0$ ).

Remark 3.1. $A_{0}$ is essentially self-adjoint on $\mathscr{D}$ in $\mathscr{H}_{0}$ and its closure (also denoted by $A_{0}$ ) has the domain $D\left(A_{0}\right)=H^{2} \times H^{1}$.

Remark 3.2. The spectrum of $A_{0}$ in $\mathscr{H}_{0}$ satisfies $\sigma\left(A_{0}\right)=\sigma_{\mathrm{e}}\left(A_{0}\right)$ $\approx \sigma_{\text {a.c. }}\left(A_{0}\right)=(-\infty,-m] \cup[m, \infty)$. Here "e" stands for essential and "a.c." for absolutely continuous.

Remark 3.3. The unitary one-parameter group $e^{-i A_{0} t}$ maps $\mathscr{D}$ into $\mathscr{D}$.

Remarks 3.1 and 3.2 are easily verified by Fourier transformation and 3.3 follows from the finite propagation velocity of solutions to Eq. (1.1) in the free case. 
Theorem 3.4. The operator $A$ is essentially self-adjoint on $\mathscr{D}$ in $\mathscr{H}$ and its closure (also denoted by $A$ ) has the domain $D(A)=D\left(A_{0}\right)=H^{2} \times H^{1}$. The essential spectrum of $A$ and $A_{0}$ coincide, $\sigma_{\mathrm{e}}(A)=\sigma_{\mathrm{e}}\left(A_{0}\right)$.

Proof. We have seen that $A=A_{0}+V$ is symmetric on $\mathscr{D}$ in $\mathscr{H}$ and that $A_{0}$ is essentially self-adjoint on $\mathscr{D}$ in $\mathscr{H}_{0}$. It then follows from a theorem due to Thoe (see Appendix 1) that the condition

$$
\|V f\|_{0} \leqq \beta\left\|A_{0} f\right\|_{0}+\gamma\|f\|_{0}, \quad \beta<1, \quad f \in \mathscr{D},
$$

ensures the essential self-adjointness of $A$ on $\mathscr{D}$ in $\mathscr{H}$. We will in fact show that $\beta$ can be chosen arbitrarily small. It is sufficient for (3.3) to hold that

$$
\|V f\|_{0}^{2} \leqq \beta^{\prime}\left\|A_{0} f\right\|_{0}^{2}+\gamma^{\prime}\|f\|_{0}^{2},
$$

where $\beta^{\prime}$ can be chosen arbitrarily small. The estimate (3.4) follows from the following estimates ${ }^{1}\left(q(x) \in L^{2}\left(R^{3}\right), q_{0}(x) \in L^{4}\left(R^{3}\right)\right)$

and

$$
\left\|q f_{1}\right\|_{2}^{2} \leqq \varepsilon_{1}\left\|L_{0} f_{1}\right\|_{2}^{2}+\delta_{1}\left(\varepsilon_{1}\right)\left\|f_{1}\right\|_{2}^{2},
$$

where $\varepsilon_{1}, \varepsilon_{2}>0$ are arbitrary (see [9], p. 302 and p. 321). This finishes the proof of the essential self-adjointness of $A$ on $\mathscr{D}$ in $\mathscr{H}$.

In order to ensure that $\sigma_{\mathrm{e}}(A)=\sigma_{\mathrm{e}}\left(A_{0}\right)$ it is sufficient to show that $V$ is a compact operator from $D\left(A_{0}\right)$ to $\mathscr{H}_{0}$ (see [10], p. 18) or, equivalently that $q\left(q_{0}\right)$ is a compact operator from $H^{2}\left(H^{1}\right)$ to $H^{0}$. This, however follows from conditions ii) and iii) on $q_{0}(x)$ and $q_{s}(x)$ (see [10], p. 104).

\section{Eigenfunction Expansions Associated with $\boldsymbol{A}$ and the Absolutely Continuous Spectrum $\sigma_{\text {a.c. }}(A)$}

In this section we construct eigenfunctions and generalized eigenfunctions of $A$. These constitute a particular set of weak solutions to the "eigenvalue equation"

$$
A \Phi=\omega \Phi, \quad \omega \in \sigma(A) .
$$

Equation (4.1) can be written

$$
\left(\omega^{2}-2 \omega q_{0}-L\right) u=0,
$$

with $\Phi=\{u, \omega u\}$, and is of Schrödinger type. This means that we can make use of the well-known properties of solutions to the timeindependent Schrödinger equation (see [5-8]).

Let us define

$$
L(\omega)=L+2 \omega q_{0} .
$$

${ }^{1}$ The lower index " 2 " denotes $L^{2}\left(R^{3}\right)$. 
Remark 4.1. The Schrödinger operator $L(\omega), \omega \in R$ is self-adjoint on $D(L(\omega))=H^{2}$ in $L^{2}\left(R^{3}\right)$ with $\sigma_{\mathrm{e}}(L(\omega))=\sigma_{\text {a.c. }}(L(\omega))=\left[m^{2}, \infty\right), L(\omega)$ has a finite number of eigenvalues (see [10], p. 218) and $L(0)$ is positive (follows from condition iv) on the potentials).

Let $u_{n}$ denote a square integrable solution of Eq. (4.2) with $\omega=\omega_{n} \in R$. Remark 4.1 implies that $u_{n} \in H^{2}$ and that $\left|\omega_{n}\right|<m$.

Remark 4.2. The eigenfunctions $\Phi_{n}$ of $A$ have the form $\Phi_{n}=\left\{u_{n}, \omega_{n} u_{n}\right\}$, i.e. $A \Phi_{n}=\omega_{n} \Phi_{n}$ with $\Phi_{n} \in D(A)$. We assume $\Phi_{n}$ to be normalized such that $\left(\Phi_{n}, \Phi_{m}\right)=\delta_{n m}$.

Remark 4.2 and the fact that $\sigma_{\mathrm{e}}(A)=(-\infty,-m] \cup[m, \infty)$, implies that the projection operator $P_{d}$ given by

$$
P_{d}=E(m)-E(-m)=\int_{-m}^{m} d E(\lambda)
$$

(with $\left.A=\int_{-\infty}^{\infty} \lambda d E(\lambda)\right)$ has the following representation

$$
P_{d} f=\sum_{n} \Phi_{n}\left(f, \Phi_{n}\right) \equiv \sum_{n} \Phi_{n} \hat{f}_{n}, \quad f \in \mathscr{H},
$$

i.e. every element in $P_{d} \mathscr{H}$ can be expanded in terms of $\left\{\Phi_{n}\right\}$.

Let us introduce

and

$$
P^{+}=\int_{m}^{\infty} d E(\lambda), \quad P^{-}=\int_{-\infty}^{-m} d E(\lambda), \quad P=P^{+}+P^{-},
$$

$$
\mathscr{H}^{ \pm}=P^{ \pm} \mathscr{H}, \quad A^{ \pm}=P^{ \pm} A .
$$

Our goal is to obtain a formula similar to (4.5) for $P^{ \pm} f$.

It is well-known that Eq. (4.2), under our conditions on the potentials, has solutions which satisfy the following integral equation (LippmannSchwinger equation) (see [5])

$u^{ \pm}(x, k)=e^{i k x}-\frac{1}{4 \pi} \int d y \frac{e^{ \pm i|k||x-y|}}{|x-y|}\left( \pm 2 \omega(k) q_{0}(y)+q(y)\right) u^{ \pm}(y, k)$,

where $\omega(k)=\sqrt{k^{2}+m^{2}}$.

Remark 4.3. A solution $u^{ \pm}(x, k)$ of Eq. (4.8) has the following properties; a) it is unique, b) it is bounded on $R^{3} \times K$, c) $u^{ \pm}(x, k)-e^{i k x}$ is uniformly continuous on $R^{3} \times K$. Here $K$ is a compact set in $R^{3}-\{0\}$. (For the proof see [5].)

Remark 4.4. The operator $A$ has as a generalized eigenfunction $\Phi^{ \pm}(x, k)=c_{k}\left\{u^{ \pm}(x, k), \pm \omega(k) u^{ \pm}(x, k)\right\}$. This is easily seen to be a weak solution of Eq. (4.1) with $\omega= \pm \omega(k)$. We choose $c_{k}=\frac{1}{\sqrt{2}(2 \pi)^{3 / 2} \omega(k)}$. 
Let $(,)_{N}$ denote the scalar product in $\mathscr{H}$ when the integration is only carried out over a sphere of radius $N$ in $R^{3}$.

Theorem 4.5. The mapping $F^{ \pm} ; \mathscr{H} \rightarrow L^{2}\left(R^{3}\right)$ defined by

$$
F^{ \pm} f(k)=\lim _{N \rightarrow \infty}\left(f, \Phi^{ \pm}(\cdot, k)\right)_{N}=\hat{f}^{ \pm}(k),
$$

is isometric with initial domain $\mathscr{H}^{ \pm}$and the whole of $L^{2}\left(R^{3}\right)$ as range.

The adjoint $F^{ \pm *}$ is given by

$$
F^{ \pm *} g(x)=\lim _{n \rightarrow \infty} \int_{K_{n}} d k \Phi^{ \pm}(x, k) g(k),
$$

where $K_{n} \subset R^{3}$ is an increasing set of compacts such that $U K_{n}=R^{3}$.

The operator $F^{ \pm}$diagonalizes $A^{ \pm}$;

$$
A^{ \pm}= \pm F^{ \pm *} M_{\omega(\cdot)} F^{ \pm},
$$

where $M_{\omega(k)}$ stands for the multiplication operator $\omega(k)$.

The singular spectrum of $A$ fulfills $\sigma_{\mathrm{s}}(A) \subset(-m, m)$, i.e. $\sigma_{\text {a.c. }}(A)=\sigma_{\mathrm{e}}(A)$.

Remark 4.6. The analog to formula (4.5) reads $P^{ \pm} f=F^{ \pm *} F^{ \pm} f$.

Proof of Theorem 4.5. We shall start by showing that $F^{ \pm}$is isometric with initial domain $\mathscr{H}^{ \pm}$. Our proof is based on the following formula

$$
\begin{aligned}
I\left(\lambda, \lambda^{\prime}, f\right) & =\left(\left(E(\lambda)-E\left(\lambda^{\prime}\right)\right) f, f\right) \\
& =\lim _{\varepsilon \downarrow 0} \frac{1}{2 \pi i} \int_{\lambda^{\prime}}^{\lambda} d \mu((R(\mu+i \varepsilon)-R(\mu-i \varepsilon)) f, f),
\end{aligned}
$$

where $R(z)=(A-z)^{-1}, \sigma_{\mathrm{s}}(A) \cap\left(\lambda^{\prime}, \lambda\right)=\phi$, and $f \in \mathscr{D}$.

The right hand side of Eq. (4.12) will now be evaluated in a series of lemmas and takes the form

$$
I\left(\lambda, \lambda^{\prime}, f\right)=\int_{\lambda^{\prime}<\omega(k)<\lambda} d k\left|F^{ \pm} f(k)\right|^{2},
$$

where the \pm sign is chosen when ${ }_{-\infty}^{m}<\lambda^{\prime}<\lambda<{ }_{-m}^{\infty}$. The integral $I\left(\lambda, \lambda^{\prime}, f\right)$ turns out to be continuous as a function of $\lambda$ and $\lambda^{\prime}$ in the interval given above, which implies that $\sigma_{\mathrm{s}}(A) \subset(-m, m)$ and furthermore that $F^{ \pm}$maps $\mathscr{H}^{ \pm}$isometrically into $L^{2}\left(R^{3}\right)$.

Lemma 4.6. Let $f \in \mathscr{D}$, then

where

$$
R(z) f=\frac{1}{z}\{1, z\} G(z)\left(L f_{1}+z f_{2}\right)-\frac{1}{z}\left\{f_{1}, 0\right\}, \quad \operatorname{Im} z \neq 0,
$$

$$
G(z)=\left(L+2 z q_{0}-z^{2}\right)^{-1},
$$

is of the Carleman type in $L^{2}\left(R^{3}\right)$ for $\operatorname{Im} z \neq 0$. 
Proof. The operator $G(z)$ is easily seen to exist and to be bounded in $L^{2}\left(R^{3}\right)$ for $z$ sufficiently small (due to the positivity of $L$ and the $L$-boundedness of $q_{0}$ ). Formula (4.14) can than be verified for small $z$ by straightforward algebra and the analyticity of $R(z) f$ in $\mathscr{H}$ can be employed to extend the validity of Eq. (4.14) to all $z$ such that $\operatorname{Im} z \neq 0$.

The first resolvant equation for $G(z)$ has the form

$$
G(z)=G_{0}(z)-G(z)\left(2 z q_{0}+q\right) G_{0}(z), \quad \operatorname{Im} z \neq 0,
$$

where $G_{0}(z)=\left(L_{0}-z^{2}\right)^{-1}$. The operator $G_{0}(z)$ is of the Carleman type and $\left(2 z q_{0}+q\right) G_{0}(z)$ is compact in $L^{2}\left(R^{3}\right)$ for $\operatorname{Im} z \neq 0$; thus the boundedness of $G(z)$ in $L^{2}\left(R^{3}\right)$ (which follows from Eq. (4.14)) and Eq. (4.16) implies that $G(z)$ is of the Carleman type for $\operatorname{Im} z \neq 0$ (for more details see [11]). This finishes the proof of the lemma.

Remark 4.7. Let $g \in H^{2} \times L^{2}\left(R^{3}\right)$ and $h \in \mathscr{H}$, then

$$
(g, h)=\left(L g_{1}, h_{1}\right)_{2}+\left(g_{2}, h_{2}\right)_{2} .
$$

Lemma 4.8. The kernel $G(x, y ; z)$ of $G(z)$ satisfies

$$
|G(x, y ; \mu-i 0)| \leqq C_{1}\left(\frac{1}{|x-y|}+C_{2}\right), \quad \mu \in \sigma_{\mathrm{e}}(A)-\{ \pm m\} .
$$

For the proof see Ikebe [5].

The second resolvant equation for $G(z)$ has the form

$$
G(z)-G\left(z^{\prime}\right)=\left(z-z^{\prime}\right) G(z)\left(z+z^{\prime}-2 q_{0}\right) G\left(z^{\prime}\right),
$$

and by combining Eqs. (4.15), (4.17), (4.18) and (4.19) one gets

$$
I\left(\lambda, \lambda^{\prime}, f\right)=\lim _{\varepsilon \downarrow 0} \frac{2 \varepsilon}{\pi} \int_{\lambda^{\prime}}^{\lambda} d \mu\left\|G(\mu-i \varepsilon) f_{\mu}\right\|_{2}^{2},
$$

where $f_{\mu}=L f_{1}+\mu f_{2}$. The estimate (4.18) is used to show that the last term in Eq. (4.19) does not survive in the limit $\varepsilon \downarrow 0$ (for details see [11]).

Lemma 4.9. A solution Eq. (4.8) is related to the kernel of $G(z)$ by the following formula

$$
\begin{gathered}
\lim _{\varepsilon \downarrow 0}\left(|k|^{2}+( \pm \omega(k)+i \varepsilon)^{2}+m^{2}\right) \int d y G(x, y ; \pm \omega(k)+i \varepsilon) e^{i k y} \\
=u^{ \pm}(x, k) \text { on } R^{3} \times K .
\end{gathered}
$$

Lemma 4.9 is proved in complete analogy with Lemma 9.2 in Ikebe [5].

Eq. (4.13) can now be verified by inserting Eq. (4.21) into Eq. (4.20), using the Parseval equality and finally employing the following formal identity

$$
\lim _{\varepsilon \downarrow 0} \frac{1}{\pi} \frac{\varepsilon}{\left(\omega(k)^{2}-\mu^{2}+\varepsilon^{2}\right)^{2}+4 \mu^{2} \varepsilon^{2}}=\frac{1}{2 \mu} \delta\left(\omega(\mathrm{k})^{2}-\mu^{2}\right),
$$


(Remark 4.3 and the fact that $f_{\mu}$ has compact support allows us to freely interchange the order of intergrations, and also to interchange the limit with the integrations.)

It then remains to prove that $F^{ \pm}$maps onto $L^{2}\left(R^{3}\right)$ and that Eqs. (4.10) and (4.11) hold. Eq. (4.10) is easily verified by using the isometric character of $F^{ \pm}$and the definition of the adjoint $\left(F^{ \pm} f, g\right)_{2}=\left(f, F^{ \pm *} g\right), f \in \mathscr{H}$, $g \in L^{2}\left(R^{3}\right)$. Eq. (4.11) is proved as follows: Let $f^{ \pm}=P^{ \pm} f$, with $f \in D(A)$; the spectral representation for $A$ together with Eqs. (4.12) and (4.13) then gives

$$
\begin{aligned}
\left(A f^{ \pm}, f^{ \pm}\right) & =\int \lambda d\left(E(\lambda) f^{ \pm}, f^{ \pm}\right)=\int d k \pm \omega(k)\left|F^{ \pm} f(k)\right|^{2} \\
& = \pm\left(M_{\omega(\cdot)} F^{ \pm} f, F^{ \pm} f\right)_{2}= \pm\left(F^{ \pm *} M_{\omega(\cdot)} F^{ \pm} f, f\right) .
\end{aligned}
$$

The ontoness of $F^{ \pm}$is verified by showing that $F^{ \pm *}$ has a trivial nullspace. We follow a similar proof, given in the Schrödinger case, by Alsholm and Schmidt [8].

Lemma 4.10. Let $g_{1}, g_{2} \in L^{2}\left(R^{3}\right)$ and let $\chi_{i}$ be characteristic functions corresponding to sets $\left\{k ; \omega(k) \in I_{i}\right\}$, where $I_{1}$ and $I_{2}$ are disjoint intervals on $R^{+}$; then

$$
\left(F^{ \pm *} \chi_{1} g_{1}, F^{ \pm *} \chi_{2} g_{2}\right)=0 \text {. }
$$

Proof. See Appendix 2.

Let us assume that $F^{+*} g=0$. Lemma 4.10 and Eq. (4.10) then give

$$
\int_{\lambda^{\prime}<\omega(k)<\lambda} d k u^{+}(x, k) g(k)=0, \quad m<\lambda^{\prime}<\lambda,
$$

and

$$
\int_{|\Omega|=1} d \Omega u^{+}(x,|k| \Omega) g(|k| \Omega)=0 \text { for a.e. }|k|>0 .
$$

The Lippmann-Schwinger equation (4.8) finally gives

$$
\int_{|\Omega|=1} d \Omega e^{i|k| \Omega x} g(|k| \Omega)=0, \text { for a.e. }|k|>0 .
$$

But this shows that $g=0$, due to the Fourier inversion theorem. The same conclusion holds of course for $F^{-*}$. The proof of Theorem 4.5 is thus completed.

\section{Existence and Completeness of the Wave-Operators $W_{ \pm}$and an Explicit Representation of the $S$-Matrix}

In scattering theory one is concerned with the asymptotic behaviour of solutions to some evolution equation for large positive and negative times. 
We shall in this section study the asymptotic behaviour of solutions to the Klein-Gordon equation (1.1) or, equivalently Eq. (2.4) $i \partial_{t} \Psi=A \Psi$, with $\Psi=\left\{u, i \partial_{t} u\right\}$. We shall in fact show the existence of two waveoperators $W_{ \pm}$which have the property that

$$
e^{-i A t} W_{ \pm} f \rightarrow e^{-i A_{0} t} f, \quad t \rightarrow \pm \infty, f \in \mathscr{H}_{0} .
$$

Our results are collected in the following theorem:

Theorem 5.1. a) The wave-operators $W_{ \pm}$defined by

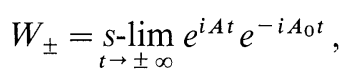

exist as isometric mappings of $\mathscr{H}_{0}$ into $P \mathscr{H}$ and they intertwine $A$ and $A_{0}$, $A W_{ \pm} \supset W_{ \pm} A_{0}$.

b) One has

$$
P^{ \pm} W_{-}=F^{ \pm *} F_{0}^{ \pm}, \quad W_{+} f=\overline{W_{-} \bar{f}}
$$

i.e. $W_{ \pm}$maps onto $P \mathscr{H}$.

c) The scattering operator $S$ defined by

$$
S=W_{+}^{*} W_{-} P_{0}^{+}+W_{-}^{*} W_{+} P_{0}^{-},
$$

is unitary in $\mathscr{H}_{0}$ and commutes with $A_{0}$.

The following representation for $\hat{S}^{ \pm}=F_{0}^{ \pm} S F_{0}^{ \pm *}$ holds

where

$$
\hat{S}^{ \pm} g(|k| \Omega)=g(|k| \Omega)-\pi i|k| \int d \Omega^{\prime} T^{ \pm}\left(|k|, \Omega, \Omega^{\prime}\right) g\left(|k| \Omega^{\prime}\right),
$$

$T^{ \pm}\left(|k|, \Omega, \Omega^{\prime}\right)=\frac{1}{(2 \pi)^{3}} \int d x e^{-i|k| \Omega^{\prime} x}\left( \pm 2 \omega(k) q_{0}(x)+q(x)\right) u_{ \pm}(x,|k| \Omega),(5.6)$ with $u_{+}(x, k)=u^{+}(x, k)$ and $u_{-}(x, k)=\overline{u^{-}(x,-k)}$.

Remark 5.2. $T^{ \pm}\left(|k|, \Omega, \Omega^{\prime}\right)$ is the phase factor appearing in the asymptotic expansion of $u_{ \pm}(x, k)$ for large $|x|$,

$$
u_{ \pm}(x, k)=e^{i k x}+2 \pi^{2} T^{ \pm}\left(|k|, \frac{x}{|x|}, \frac{k}{|k|}\right) \frac{e^{i|k||x|}}{|x|}+\mathcal{O}\left(\frac{1}{x}\right) .
$$

The proof of Theorem 5.1 will be divided into three parts. First we show the existence of $W_{ \pm}$as defined in Eq. (5.2), then Eq. (5.3) is established, and finally Eq. (5.6) is derived.

Proof of Theorem 5.1. a) By differentiating and integrating $W(t)=e^{i A t} e^{-i A_{0} t}$ we get

and thus

$$
W(t) f=f+i \int_{0}^{t} d t e^{i A t} V e^{-i A_{0} t} f, \quad f \in \mathscr{D} \subset D(V),
$$

$$
\|W(t) f\| \leqq\|f\|+\int_{0}^{t} d t\left\|V e^{-i A_{0} t} f\right\|,
$$


which shows that it is sufficient for the existence of $W_{ \pm} f$ that $\left\|V e^{-i A_{0} t} f\right\|$ is integrable on $(0, \pm \infty)$.

We have

$$
\left\|V e^{-i A_{0} t} f\right\|^{2}=\left\|q f_{1}(t)\right\|_{2}^{2}+\left\|2 q_{0} f_{2}(t)\right\|_{2}^{2},
$$

where $f(t)=\left\{f_{1}(t), f_{2}(t)\right\}=e^{-i A_{0} t} f$.

Let $\Theta_{N}$ denote the characteristic function for a sphere of radius $N$ around the origin in $R^{3}$.

Lemma 5.2. The following estimate holds

$$
\left\|\Theta_{N} f_{i}(t)\right\|_{\infty} \leqq \text { const }|t|^{-3 / 2}, \quad|t| \geqq 4 N, \quad i=1,2,
$$

where the constant is independent of $N$.

For the proof see [11], Lemma 8.1, or [12] where a similar result is proved in the Dirac case.

We are now in the position to estimate the right-hand side of Eq. (5.10). The first term is estimated as follows $(t=4 N)$;

$$
\begin{aligned}
\left\|q f_{1}(t)\right\|_{2} & \leqq\left\|\left(1-\Theta_{N}\right) q f_{1}(t)\right\|_{2}+\left\|\Theta_{N} q f_{1}(t)\right\|_{2} \\
& \leqq\left\|\left(1-\Theta_{N}\right) q\right\|_{\infty}\left\|f_{1}(t)\right\|_{2}+\|q\|_{2}\left\|\Theta_{N} f_{1}(t)\right\|_{\infty} \\
& =\mathcal{O}\left(|t|^{-3 / 2}\right),
\end{aligned}
$$

where we have used the fact that $q(x)=\mathcal{O}\left(|x|^{-3-\varepsilon}\right),|x| \rightarrow \infty$, and Lemma 5.2. We get a similar result for $\left\|q_{0} f_{1}(t)\right\|_{2}$.

This shows that $\left\|V e^{i A_{0} t} f\right\|$ is integrable and thus $W_{ \pm}$exists on $\mathscr{D}$. One can then easily verify that $\left\|W_{ \pm} f\right\|=\|f\|_{0}$ by observing that $\|f\|^{2}=\|f\|_{0}^{2}+\left(q f_{1}, f_{1}\right)_{2}$ and using an estimate similar to (5.12).

The domain of $W_{+}$can now be extended by continuity to the whole of $\mathscr{H}_{0}$ and we furthermore have $P W_{ \pm}=W_{ \pm}$and $A W_{ \pm} \supset W_{ \pm} A_{0}$ (see Kato [13], p. 346). This completes the proof of a) in Theorem 5.1.

b) In this part of the proof we use a different but equivalent definition of $W_{ \pm}$.

Lemma 5.3. One has

$$
W_{ \pm}=W_{ \pm}(J)=s-\lim _{t \rightarrow \pm \infty} e^{i A t} J e^{-i A_{0} t},
$$

where $J$ is defined by $(J f, g)=(f, g)_{0}, f, g \in \mathscr{H}$.

Proof. See Appendix 3.

We now show that $P^{ \pm} W_{-}=F^{ \pm *} F_{0}^{ \pm}$. Put $W_{J}(t)=e^{i A t} J e^{-i A_{0} t}$, $B(t)=F_{0}^{+*} F^{+} W_{J}(t)$, and choose $f$ and $g$ such that $f \in \mathscr{D}$ and $\hat{g}_{0}^{+} \in C_{0}^{\infty}\left(R^{3}-\{0\}\right)$ [one can easily verify that this means that $\left.g \in D(A)\right]$. 
Let us consider $(B(t) f, g)_{0}$. By differentiating and integrating it we get

$$
(B(t) f, g)_{0}=\left(F_{0}^{+*} F^{+} J f, g\right)_{0}+i \int_{0}^{t} d t\left(F_{0}^{+*} F^{+} e^{i A t} V^{\prime} e^{-i A_{0} t} f, g\right)_{0},
$$

where $V^{\prime}=A J-J A_{0}$. The integrand can be rearranged as follows (we put $F=F^{+}$and $F_{0}=F_{0}^{+}$),

$$
\begin{aligned}
\left(F_{0}^{*} F e^{i A t}\right. & \left.V^{\prime} e^{-i A_{0} t} f, g\right)_{0}=\left(V^{\prime} e^{-i A_{0} t} f, e^{-i A t} F^{*} F_{0} g\right) \\
& =\left(e^{-i A_{0} t} f, V e^{-i A t} F^{*} F_{0} g\right)_{0}=\left(e^{-i A_{0} t} f, V F^{*} e^{-i \omega(\cdot) t} F_{0} g\right)_{0} \\
& =\left(e^{-i A_{0} t} f, V \int d k \Phi^{+}(\cdot, k) e^{-i \omega(k) t} \hat{g}_{0}^{+}(k)\right)_{0} \\
& =\int d k\left(f, e^{i\left(A_{0}-\omega(k)\right) t} V \Phi^{+}(\cdot, k)\right)_{0} \frac{\hat{g}_{0}^{+}(k)}{}
\end{aligned}
$$

where we have used the definition of $J$ and interchanged the order of integrations, which is allowed due to the absolute convergence of the integrals $\left(H^{+}(\cdot, k)=V \Phi^{+}(\cdot, k) \in \mathscr{H}_{0}\right)$.

Equations (5.2) and (5.13) ensures the existence of the limit as $t \rightarrow-\infty$ of Eq. (5.14); thus we are allowed to take the Abelian limit and get

$$
\begin{aligned}
\left(F_{0}^{+*} F^{+} W_{-} f, g\right)_{0}= & \int d k\left(f, \Phi^{+}(\cdot, k)\right)_{0} \overline{\hat{g}_{0}^{+}(k)} \\
& +\lim _{\varepsilon \downarrow 0} \int d k\left(f, R_{0}(\omega(k)+i \varepsilon) H^{+}(\cdot, k)\right)_{0} \overline{\hat{g}}_{0}^{+}(k)
\end{aligned}
$$

The Lippmann-Schwinger Equation (4.8) can be written

$$
\Phi^{ \pm}(\cdot, k)=\Phi_{0}^{ \pm}(\cdot, k)-\left(R_{0}( \pm \omega(k)+i 0) V\right) \Phi^{ \pm}(\cdot, k),
$$

where $\Phi_{0}^{ \pm}(\cdot, k) \in L^{\infty}\left(R^{3}\right) \times L^{\infty}\left(R^{3}\right) \equiv\left(L^{\infty}\right)^{2}$ and $\left(R_{0}( \pm \omega(k)+i 0) V\right)$ is a compact integral operator on $\left(L^{\infty}\right)^{2}$ (compare [5], p. 14).

By inserting Eq. (5.17) into Eq. (5.16) we finally get

$$
\left(F_{0}^{+*} F^{+} W_{-} f, g\right)_{0}=\int d k\left(f, \Phi_{0}^{+}(\cdot, k)\right)_{0} \overline{\hat{g}_{0}^{+}(k)}=\left(P_{0}^{+} f, g\right)_{0},
$$

which implies that

and thus

$$
\begin{aligned}
& F_{0}^{+*} F^{+} W_{-}=P_{0}^{+}, \\
& P^{+} W_{-}=F^{+*} F_{0}^{+} .
\end{aligned}
$$

In a similar way one shows that $P^{-} W_{-}=F^{-*} F_{0}^{-}$and it is furthermore simple to verify that $W_{+} f=\overline{W_{-} \bar{f}}$. This completes the proof of part b) of Theorem 5.1.

c) Let $f \in D\left(A_{0}\right) \subset D(V)$; we then get

$$
W_{+} f-W_{-} f=i \int_{-\infty}^{\infty} d t e^{i A t} V e^{-i A_{0} t} f,
$$

and by applying $W_{+}^{*}$ we get

$$
W_{+}^{*} W_{-} f=f-i \int_{-\infty}^{\infty} d t e^{i A_{0} t} W_{+} V e^{-i A_{0} t} f
$$


Let $f, g \in \mathscr{H}_{0}^{+}$be such that $\hat{f}_{0}^{+}, \hat{g}_{0}^{+} \in C_{0}^{\infty}\left(R^{3}-\{0\}\right),\left(f, g \in D\left(A_{0}\right)\right)$. By inserting Eq. (5.22) into Eq. (5.4) we obtain

$$
\begin{aligned}
(S f, g)_{0} & =(f, g)_{0}-i \int_{-\infty}^{\infty} d t\left(e^{i A_{0} t} W_{+} V e^{-i A_{0} t} f, g\right)_{0} \\
& =(f, g)_{0}-i \int_{-\infty}^{\infty} d t\left(V e^{-i A_{0} t} f, W_{-} e^{-i A_{0} t} g\right) .
\end{aligned}
$$

In complete analogy with the Schrödinger case (see Ikebe [7]) we get

$$
\begin{aligned}
&(S f, g)_{0}=(f, g)_{0}- 2 \pi i \int d k d k^{\prime} \delta\left(\omega\left(k^{\prime}\right)-\omega(k)\right) \\
& \cdot\left(V \Phi_{0}^{+}(\cdot, k), \Phi^{+}\left(\cdot,-k^{\prime}\right)\right) \hat{f}_{0}^{+}(k) \overline{\hat{g}_{0}^{+}\left(k^{\prime}\right),}
\end{aligned}
$$

when $f, g \in \mathscr{H}_{0}^{+}$. In the case when $f, g \in \mathscr{H}_{0}^{-}$we obtain similarily

$$
\begin{aligned}
&(S f, g)_{0}=(f, g)_{0}-2 \pi i \int d k d k^{\prime} \delta\left(\omega\left(k^{\prime}\right)-\omega(k)\right) \\
& \cdot\left(V \Phi_{0}^{-}(\cdot, k), \Phi^{-}\left(\cdot, k^{\prime}\right)\right) \hat{f}_{0}^{-}(k) \overline{\hat{g}_{0}^{-}\left(k^{\prime}\right)} .
\end{aligned}
$$

Equation (5.5) follows directly from Eqs. (5.24) and (5.25), which completes the proof of Theorem 5.1.

\section{Summary and Conclusions}

We have in this paper developed the spectral and scattering theory for the Klein-Gordon equation with two static external potentials (coupled like a zeroth component of a four-vector and a scalar, respectively)

$$
\left(\square+2 i q_{0}(x) \partial_{t}-q_{0}(x)^{2}+q_{s}(x)+m^{2}\right) u(x, t)=0 .
$$

Our main results, which are contained in Theorems 4.5 and 5.1 are similar to those obtained in the Schrödinger case (see [8]). The main difference is that we have to impose a limit of the strength of $q_{0}$ and on the negative part of $q_{s}$ (see condition iv), p. 244).

Theorem 4.5 can be employed to explicitly diagonalize the quantumfield-theoretic Hamiltonian for a charged scalar field interacting with two kinds of external static fields $q_{0}(x)$ and $q_{s}(x)$. Furthermore, Theorem 5.1 can be used to prove that the $S$-matrix, defined in the LSZsense, coincides with the classical $S$-matrix [Eq. (5.5)] and that asymptotic completeness holds (under our conditions on the potentials). All this will be considered elsewhere.

Acknowledgements. I want to thank Professors L. Gårding, J. Hamilton, L. Hörmander, I. Segal and R. F. Streater for stimulating discussions. Also, I am grateful for the hospitality shown to me at Nordita. 


\section{Appendix 1}

Theorem (Thoe [1], p. 373): Let $\mathscr{V}$ be a complex linear vector space which forms a Hilbert space with respect to each of two scalar products (, ) and (, , $)_{0}$. Suppose $T_{0}$ and $V$ are closed linear operators on the Hilbert space $\mathscr{H}_{0}=\left\{\mathscr{V},(,)_{0}\right\}$, possessing the following properties:

i) $T_{0}$ is self-adjoint;

ii) $D(V) \supset D\left(T_{0}\right)$;

iii) $\|V f\|_{0} \leqq a\left\|T_{0} f\right\|_{0}+b\|f\|_{0}$, for $f \in D\left(T_{0}\right)$ and $0<a<1$;

iv) The operator $T=T_{0}+V$ with domain $D(T)=D\left(T_{0}\right)$ is symmetric in the Hilbert space $\mathscr{H}=\{\mathscr{V},()$,$\} .$

Then $T$ is self-adjoint in $\mathscr{H}$.

\section{Appendix 2}

Proof of Lemma 4.10. Let us start with the following lemma;

Lemma A 2. Let $h_{1}$ and $h_{2}$ be two functions in $C_{0}^{\infty}\left(R^{3}\right)$, such that the compact sets $S_{1}$ and $S_{2}$, defined by

$$
S_{i}=\left\{t \in R ; \exists k \in \operatorname{supp} h_{i} \quad \text { with } \quad \omega(k)=t\right\},
$$

are disjoint and contained in $R^{+}-\{0\}$. Then

$$
\left(F^{ \pm *} h_{1}, F^{ \pm *} h_{2}\right)=0 \text {. }
$$

We observe that Lemma 4.10 follows from Lemma A 2, since $\chi_{1} f_{1}$ and $\chi_{2} f_{2}$ can be approximated in $L^{2}\left(R^{3}\right)$ by sequences of $C_{0}^{\infty}$ functions having their support in $\left\{k ; \omega(k) \in I_{1}\right\}$ and $\left\{k ; \omega(k) \in I_{2}\right\}$, respectively.

Proof of Lemma A2. We start by showing that if $g \in C_{0}^{\infty}\left(R^{3}\right)$ has support in $R^{3}-\{0\}$, then $F^{ \pm *} g \in D\left(A^{n}\right)$ for each integer $n$, and

$$
p(A) F^{ \pm *} g=F^{ \pm *}(p( \pm \omega(\cdot)) g),
$$

for each real polynomial $p$. To this end we consider

$$
F^{ \pm *} g=\int d k \Phi^{ \pm}(\cdot, k) g(k) .
$$

It is easy [using Fubini's Theorem and our knowledge of $\left.\Phi^{ \pm}(x, k)\right]$ to see that

$$
A F^{ \pm *} g=\int d k \Phi^{ \pm}(\cdot, k)( \pm \omega(k)) g(k)=F^{ \pm *}( \pm \omega(\cdot) g),
$$

weakly. Explicitly this means that

$$
\left(A f, F^{ \pm *} g\right)=\left(f, F^{ \pm *}( \pm \omega(\cdot) g)\right),
$$

for any $f \in \mathscr{D}$ and thus for any $f \in D(A)$. Since $A$ is self-adjoint, it follows that $F^{ \pm *} g$ lies in $D(A)$ and (A 2.3) holds. By iterating this fact we get (A 2.2). 
Equation (A 2.1) is now easily established. In fact, the self-adjointness of $p(A)$ gives

$$
\left(p(A) F^{ \pm *} h_{1}, F^{ \pm *} h_{2}\right)=\left(F^{ \pm *} h_{1}, p(A) F^{ \pm *} h_{2}\right),
$$

and thus, due to (A 3.4) we get

$$
\left(F^{ \pm *}\left(p( \pm \omega(\cdot)) h_{1}\right), F^{ \pm *} h_{2}\right)=\left(F^{ \pm *} h_{1}, F^{ \pm *}\left(p( \pm \omega(\cdot)) h_{2}\right)\right) .
$$

We can now choose a function $\psi(t)$ in $C_{0}^{\infty}(R)$ such that $\psi(t)=1$ for $t \in S_{1}$ and $\psi(t)=0$ for $t \in S_{2}$. We can approximate $\psi$ uniformly on $S_{1} \cup S_{2}$ by a sequence $\left\{p_{n}\right\}$ of polynomials and obtain (A 2.1) in the limit.

\section{Appendix 3}

Proof of Lemma 5.3. Let us start by observing that $J f=\left\{L^{-1} L_{0} f_{1}, f_{2}\right\}$ when $f \in \mathscr{D}$. Let $f \in \mathscr{D}$; we then get

$$
\begin{aligned}
\left\|e^{i A t}(J-1) e^{-i A_{0} t} f\right\| & =\left\|(J-1) e^{-i A_{0} t} f\right\| \\
& =\left\|C^{-1}\left(C_{0}-C\right) e^{-i A_{0} t} f\right\|=\left\|C^{-1} Q e^{-i A_{0} t} f\right\|,
\end{aligned}
$$

where $C=\left(\begin{array}{ll}L & 0 \\ 0 & 1\end{array}\right), C_{0}=\left(\begin{array}{ll}L_{0} & 0 \\ 0 & 1\end{array}\right)$, and $Q=\left(\begin{array}{rr}-q & 0 \\ 0 & 0\end{array}\right)$. Lemma 4.7 and (A 3.1) finally gives

$$
\begin{aligned}
\left\|C^{-1} Q e^{-i A_{0} t} f\right\|^{2} & =\left(Q e^{-i A_{0} t} f, C^{-1} Q e^{-i A_{0} t} f\right)_{L^{2} \oplus L^{2}} \\
& \leqq\left\|C^{-1}\right\|_{L^{2} \oplus L^{2}}\left\|Q e^{-i A_{0} t} f\right\|_{L^{2} \oplus L^{2}}^{2} \\
& =\text { const }\left\|q f_{1}(t)\right\|_{2}^{2} \rightarrow 0,|t| \rightarrow \infty,
\end{aligned}
$$

where, in the last step, we have used Eq. (5.12).

\section{References}

1. Thoe,D.: Spectral theory for the wave-equation with a potential term. Arch. Rat. Mech. Anal. 22, 364 (1966).

2. Lax,P.D., Phillips, R.S.: Scattering Theory. New York: Academic Press 1967.

3. Strauss, W.A.: Scattering for hyperbolic equations. Trans. Am. Math. Soc. 108, 13 (1963).

4. Veselic, K.: A spectral theory for the Klein-Gordon equation with an external electrostatic potential. Nucl. Phys. A 147, 215 (1970).

5. Ikebe,T.: Eigenfunction expansions associated with Schrödinger operators and their application to scattering theory. Arch. Rat. Mech. Anal. 5, 1 (1960).

6. Ikebe,T.: Remarks on the orthogonality of eigenfunctions for the Schrödinger operator in $R^{n}$. Journ. Fac. Sci. Tokyo 17, 355 (1970).

7. Ikebe, T.: On the phase-shift formula for the scattering operator. Pac. Journ. Math. 15, 511 (1965). 
8. Alsholm,P., Schmidt, G.: Spectral and scattering theory for Schrödinger operators. Various Publication Series No. 7. Aarhus Univ. (1969).

9. Kato, T.: Perturbation theory for linear operators. Berlin-Göttingen-Heidelberg: Springer 1966.

10. Schechter, M.: Spectra of partial differential operators. Amsterdam: North-Holland 1971.

11. Lundberg,L.-E.: Spectral and scattering theory for the Klein-Gordon equation. Nordita Preprint, Sept. 1971.

12. Prosser, R. T.: Relativistic potential scattering. Journ. Math. Phys. 4, 1048 (1963).

13. Kato, T.: Scattering theory with two Hilbert spaces. Journ. Func.Anal. 1, 347 (1967).

L. E. Lundberg

NORDITA

Blegdamsvej 17

DK-2100 Kopenhagen/Denmark 
